\title{
Timeaxis Design of a Service System Growing Values of Mobility Using the M-V Model
}

\author{
Kei Kamiya ${ }^{1}$, Akira Kito ${ }^{1}$, Jaime Alvarez ${ }^{2}$, Koichiro Sato ${ }^{3}$, Hidekazu Nishimura ${ }^{4}$, \\ Yoshiyuki Matsuoka ${ }^{3}$, and Satoru Furugori ${ }^{5}$ \\ ${ }^{1}$ Graduate School of Science and Technology, Keio University, Yokohama, Japan \\ k.kamiya.0123@gmail.com, fa0kitou@hotmail.co.jp \\ ${ }^{2}$ Monterrey Institute of Technology and Higher Education, Monterrey, Mexico \\ ${ }^{3}$ Department of Mechanical Engineering, Keio University, Yokohama, Japan \\ $\{\mathrm{k}$. sato, matsuoka\}@mech. keio.ac.jp \\ ${ }^{4}$ Graduate School of System Design and Management, Keio University, Yokohama, Japan \\ h.nishimuralsdm.keio.ac.jp \\ ${ }^{5}$ Mazda R\&D Center Yokohama, Yokohama, Japan \\ furugori.s@mazda.co.jp
}

\begin{abstract}
This paper describes the design of a service system which belongs to a basic system realizing a Value Growth Mobility: a concept of next generation mobility intended to increase the different values that the user feels towards the mobility. The concept is proposed based on Timeaxis Design incorporating the viewpoint of time into the theory and methodology of design. To design this service system, the $\mathrm{M}-\mathrm{V}$ model is used. This model is an integrated model of the $\mathrm{M}$ model, which focuses on design activity, and the V model, which visualizes a system development process. As the result of design, it is proposed a system consisting of subsystems including an emotion recognition system, a camera system, and a social networking service. These subsystems provide services encouraging the growth of values through interactions between the user and the mobility system.
\end{abstract}

Keywords: Timeaxis Design, M Model, V Model, Service Design.

\section{Introduction}

Recently, design of robust and attractive products is highly demanded due to environmental degradation, aging of the population, and advancement of information society [1]-[2]. To deal with these issues, Timeaxis Design (TAD), which incorporates the viewpoint of time into the theory and methodology of design, has been proposed [3]. On the other hand, Value Growth Design (VGD) [4] is concept of design growing values of a product through usage embodying TAD. As examples of VGD, leather gloves and fountain pens create growth of perceived values by fitting to the user through usage, keeping qualities by maintenance, and developing a sense of attachment caused by long-term usage. By realizing VGD based on Value Growth Model, it is considered possible to design products adaptable to various circumstances and values that are variable over time. 
In the previous study, a Value Growth Mobility was proposed by applying VGD to mobility [5]. The Value Growth Mobility is a concept of next generation mobility, and increases different values that user feels towards the mobility, by means of a mobility system. This concept contributes to address problems surrounding mobility, such as environmental degradation, passenger accidents, and a decrease of interest in vehicle purchasing. In this research, the mobility system not only refers to the vehicle, but includes services and infrastructures surrounding the vehicle. In previous studies, the Core Module system and the service system have been obtained as basic systems, as the result of conceptual design of the Value Growth Mobility [3]. The Core Module system is installed in the vehicle, and enables to adapt the vehicle to driving circumstances and user's conditions. This system uses the algorithm of the Emergent Control System [6] that allows adjustment to various driving circumstances. By using this system, it is confirmed a $36 \%$ improvement in fuel efficiency and battery life in the simulation of assumed hybrid cars. By contrast, the service system is assumed to encourage the growth of values that user feels towards the mobility through the interaction between the user and the mobility system. However, this system has not been designed towards the development.

Against this background, this research is aiming to develop the service system encouraging the growth of values that a user feels towards the mobility. To realize the growth of values, it is necessary to consider psychological elements that user feels towards the mobility. Additionally, it is assumed that the service system will become complex and large against a backdrop of the progression of ICT or infrastructures [7]. Hence, the M-V model is used to develop the service system. The M-V model is an integrated model of the M model (Multispace design model) [8], which models design acts and knowledge for these acts by using multiple spaces, and the V model [9], which supports to develop the complex and large systems. In this paper, a basic design of the service system based on the M-V model towards the development including verification and validation is described.

\section{The M-V Model and a System Design Method Based on the M-V Model}

\subsection{The M-V Model Integrated the M Model and the V Model}

The service system is designed based on the M-V model integrating the M model, which models acts and knowledge of design by multiple spaces, and the V model, which visualizes a process of a system development.

Fig. 1 shows the M model [8]. This model is composed of thinking space describing designing, and knowledge space describing knowledge used in designing. In this model, designing is defined by induction, abduction, and deduction in thinking space based on factors in knowledge space. Thinking space is comprised of psychological space including psychological elements that a user feels towards the design objective, and physical space including physical elements that realize psychological elements. 


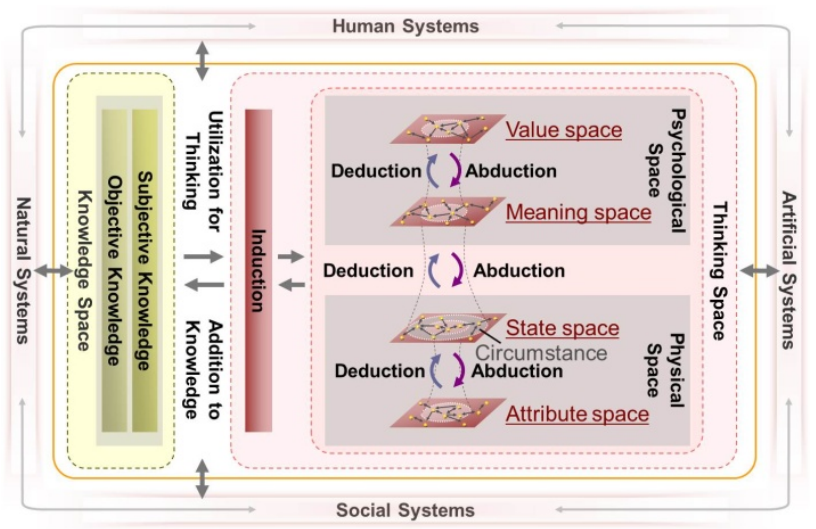

Fig. 1. M model

Psychological space consists of value space and meaning space. By contrast, physical space consists of state space and attribute space. Value space consists of numerous values such as social, cultural, and individual values. Meaning space is built of functions and images. State space includes characteristics of the system, dependent on its circumstances. Attribute space is constructed of characteristics independent of circumstances. In this model, circumstances refer to the usage environment, including the user and the ways in which artifacts are used. Knowledge space is comprised of objective knowledge and subjective knowledge. Objective knowledge holds generalities such as theories and methodologies, including physical laws in natural sciences, social sciences, and humanities. Subjective knowledge contains specialties that depend on individual contexts. When designing based on viewpoint of the $\mathrm{M}$ model, bottom-up generation of ideas caused by the extraction of elements and detection of relationships is supported. Thereby, it encourages emergent design which includes the bottom-up process. In addition, it promotes development that does not depend on apparent requirements. However, it cannot manage a whole process of development involving realization, integration, and validation.

On the other hand, the V model [9] is a valuable tool for visualizing and managing the systems engineering process. Fig. 2 shows the architecture V model. The left leg of the $\mathrm{V}$ represents system decomposition and definition of system architecture based on requirements of systems derived by analysis of stakeholder's requirements. The right leg represents realization of the lowest configuration items, architecture integration with verification of satisfaction to requirements, and validation of the system. At each decomposition level, there is a direct correlation between activities on the left and right sides of the V. For example, a test case confirming a completion of a system must be determined by an operation scenario clarified on the left. The V model can clarify each activity in a systems engineering process to multidisciplinary team. In addition, by using this model, it is possible to support the systems engineering developing solutions in response to expanded requirements by the growing scale and complexity of large systems. However, the systems engineering along the $\mathrm{V}$ model tends to develop requirements analysis and system definition based on apparent requirements in the early stage 


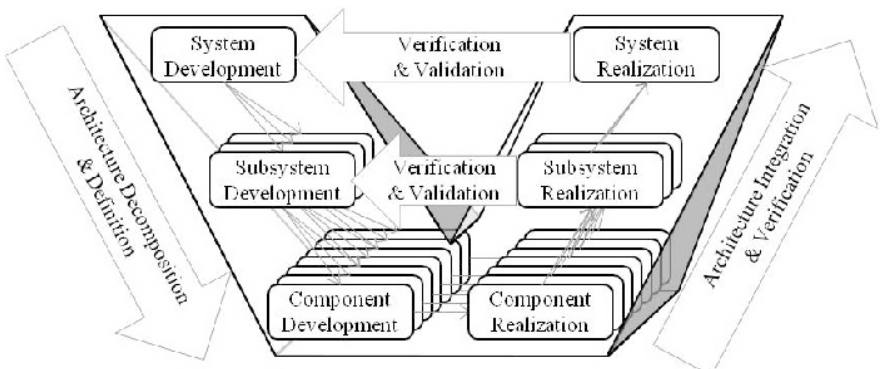

Fig. 2. Architecture V Model

of development. Thereby, there is a possibility that it is difficult to derive latent requirements. Additionally, it may be difficult to perform emergent derivation for new values in the systems engineering process due to the tendency to be top-down development in the range of determined requirements and specification. Hence, it is expected to propose the system with new values differ from existing systems by encouraging bottom-up idea generation that does not depend on apparent requirements in the stage of determining requirements or specification of systems.

There are complementary relationships between the designing based on viewpoint of the $\mathrm{M}$ model and the systems engineering along the $\mathrm{V}$ model. In the designing based on viewpoint of the $\mathrm{M}$ model, psychological elements and physical elements are organized from the viewpoint. Thereby, there are expected to realize the bottomup generation of ideas caused by the extraction of elements and the detection of relationships which do not depend on apparent requirements. This idea generation may cover the top-down design based on requirements in the early process of the systems engineering. On the other hand, in the systems engineering along the $\mathrm{V}$ model, it is possible to support the whole systems engineering process from the system requirements analysis to the latest validation. Furthermore, the systems engineering along the $\mathrm{V}$ model enables to support the verification in each stage due to ensuring traceability through the whole systems engineering process.

Based on the complementary relationships, in this research, an $\mathrm{M}-\mathrm{V}$ model integrating the $\mathrm{M}$ model and the $\mathrm{V}$ model is used to design the service system. Fig.3 shows the M-V model. This model indicates the systems engineering process along the $\mathrm{V}$ model adopting the viewpoint of the $\mathrm{M}$ model. A system design method based on the $\mathrm{M}-\mathrm{V}$ model, ensures traceability between psychological elements including value and meaning and physical elements realizing psychological elements. By using this method, the method enables to organize elements extracted in process of realization, verification, or after provision in the systems engineering process. Therefore, it is expected to perform emergent derivation of value and meaning that was not assumed in decomposition and definition process. These effects are obtained by the M$\mathrm{V}$ model integrating concepts of the $\mathrm{M}$ model and the $\mathrm{V}$ model. However, they are difficult to obtain just combining the design method based on the $\mathrm{M}$ model, which does not manage the process of verification and validation, and the systems engineering, which develops the system based on apparent requirements. 


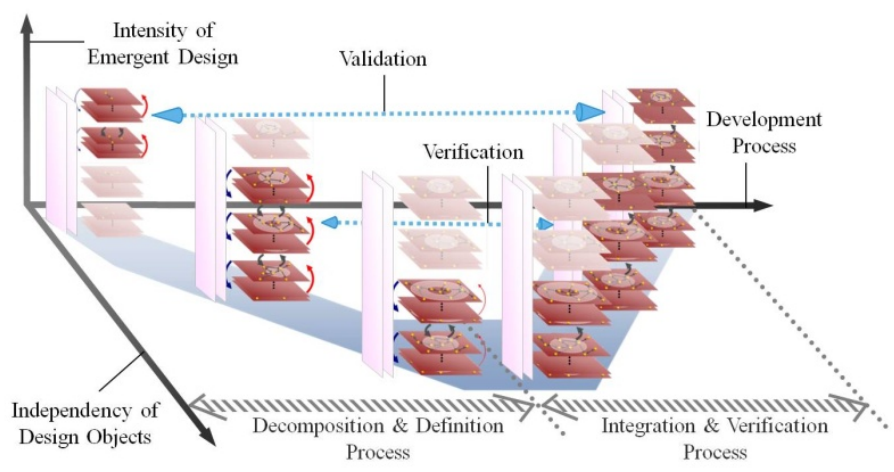

Fig. 3. M-V model

\subsection{A System Design Method Using the M Method and SysML}

To design a system based on the M-V model, the system design method combining the M method with an elements relationship diagram [10] and SysML (Systems Modeling Language) [11] is employed. The $\mathrm{M}$ method with the elements relationship diagram is a design method based on the viewpoint of the M model. On the other hand, SysML is a modeling language realizing the systems engineering along the $\mathrm{V}$ model.

The $\mathrm{M}$ method is the design method that adopts viewpoints of the M model. In this method, the system is developed by organizing elements and relationships between elements in circumstances and four types of space: value, meaning, state and attribute. In this research, the $\mathrm{M}$ method with the elements relationship diagram is used. The elements relationship diagram describes each element and their relationships included in each space of the system. This method enables to develop the design considering both psychological elements and physical elements at the same time.

On the other hand, SysML is a graphic modeling language that supports specification, design, verification, and validation of complex systems. The language describes systems, their components and environment, and supports modeling the whole system by using nine diagrams classified into four groups: requirements, behavior, parametrics and structure diagram. SysML enables the development of solutions which address expanded requirements of large and complexity systems.

By using the M method with the elements relationship diagram and SysML, it is expected to structure systems that enable to adapt changing conditions of users and circumstances over time. Fig. 4 shows the combined system design method. Here, the four steps listed below are repeated optionally.

1. Modelling elements relationship diagram by the M method

Extract elements and organize relationships between elements. In this step, design considering elements of psychology, physics, and circumstances are developed to extract and organize elements by viewpoints of multispace.

2. Translating to SysML

Translate elements and relationships to SysML. Viewpoints of the M model are included to systems engineering through SysML. For example, 


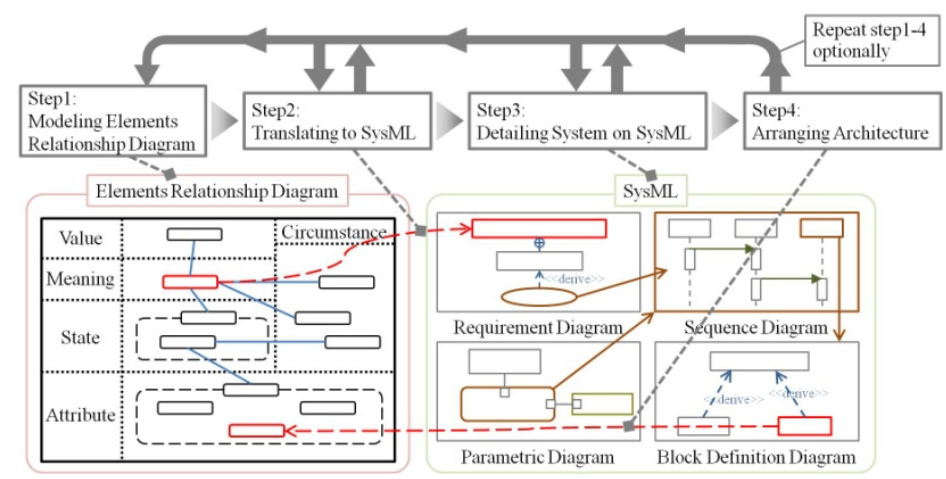

Fig. 4. Flow of Proposed System Design Method

value and meaning elements are described in SysML as the basic elements of requirements.

3. Detailing System in SysML

Detail system in SysML, along with systems engineering process. For instance, behaviours and structures of the system are newly derived from requirements based on value elements.

4. Arranging Architecture

Translate extracted elements from SysML to their elements relationship diagram, and arrange system architecture. To ensure traceability, describe in each element about relationships of it and SysML diagrams which detail it. For example, subsystems derived in SysML are arranged as attribute elements in the elements relationship diagram, and IDs of SysML diagrams that describe behavior or structure of those subsystems are put down in each attribute element.

By repeating these steps, the system architecture is modelled. In the process of realization, verification, and validation, the system is developed based on models obtained in the process of design.

\section{Proposal of the Service System Realizing the Value Growth Mobility}

\subsection{The Service System Including Viewpoint of Timeaxis Design}

The Value Growing Mobility is proposed based on Value Growth Design (VGD), which is a design concept realizing Timeaxis Design (TAD). TAD incorporates the viewpoint of time into the theory and methodology of design, enabling to adapt various circumstances and user's conditions as well as changing the two over time to be considered. VGD is proposed based on this concept of TAD. By applying the VGD to products, values, which the user feels towards the products, increase proportionally to the amount of usage. In previous studies of VGD, a Value Growth Model is proposed as a model describing changes of values over time in the process of product's value 
growth [4]. In this model, from the time before the user purchases the product to the time the user disposes it is divided by 5 phases describing different features: value discovery phase, value realization phase, value growth phase, value establishment phase, and value tradition phase. The value discovery phase occurs when the user discovers values of an object or product before purchase. The value realization phase is when, after purchase, the user uses the product or system or learns about its different functions and reconciles expectations and the actual use of a product. The value growth phase is when the user employs an object over time and develops a sense of attachment to it. The value establishment phase is when the value as a whole eventually stabilizes. The value tradition phase occurs when the value begins to decrease due to physical decay, and is transferred to the next generation of that product or system. By using viewpoints of each phase, it is considered possible to design the service system encouraging the growth of values that the user feels towards the mobility.

Based on viewpoints of the Value Growth Model, the service system realizing the Value Growth Mobility is designed by the system design method as it was explained in the previous section. The system is designed based on a concept of encouraging the growth of values that the user feels towards the mobility by making delight through travel with the vehicle. Fig. 5 shows the element relationship diagram describing key elements of the system derived from the concept. In the following sections, the service system consisted of these elements and services that they provide are explained.

\subsection{Structure of the Proposed System}

Fig. 6 shows an internal block diagram describing the structure of the proposed service system. The internal block diagram represents interconnection and interfaces between blocks. For example, Fig. 6 describes interactions between the block on the left side, "user" and blocks representing structural elements of the service system. Nested blocks within "vehicle" or "Service Department" represent subsystems of each

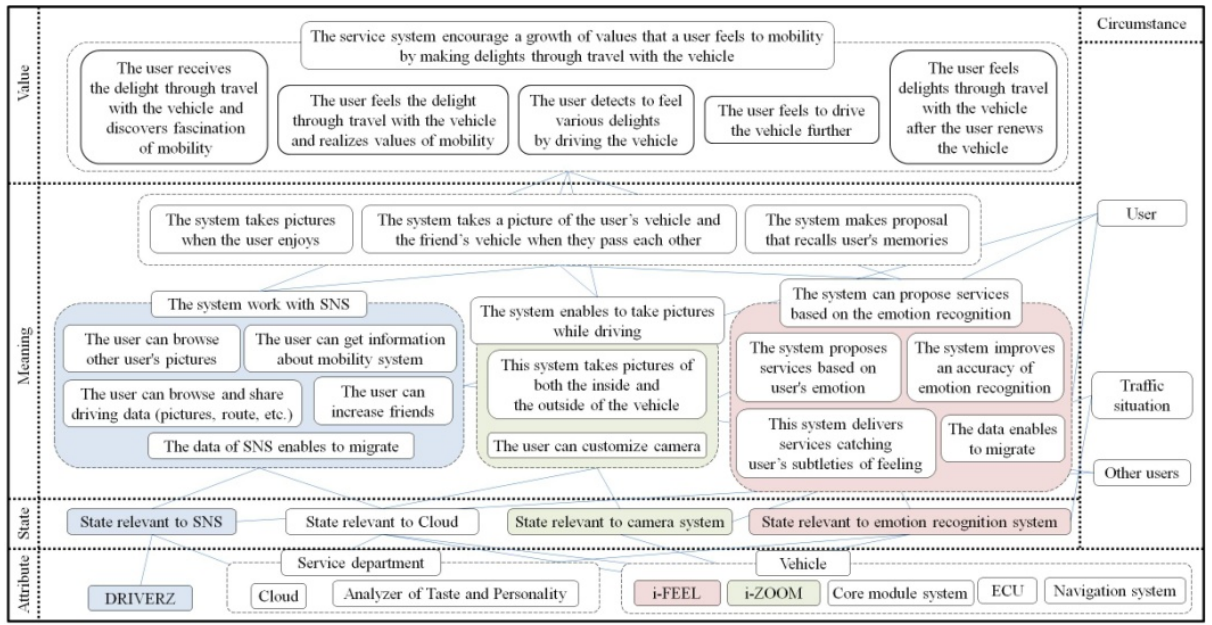

Fig. 5. Elements Relationship Diagram Describing Key Elements of the Service System 


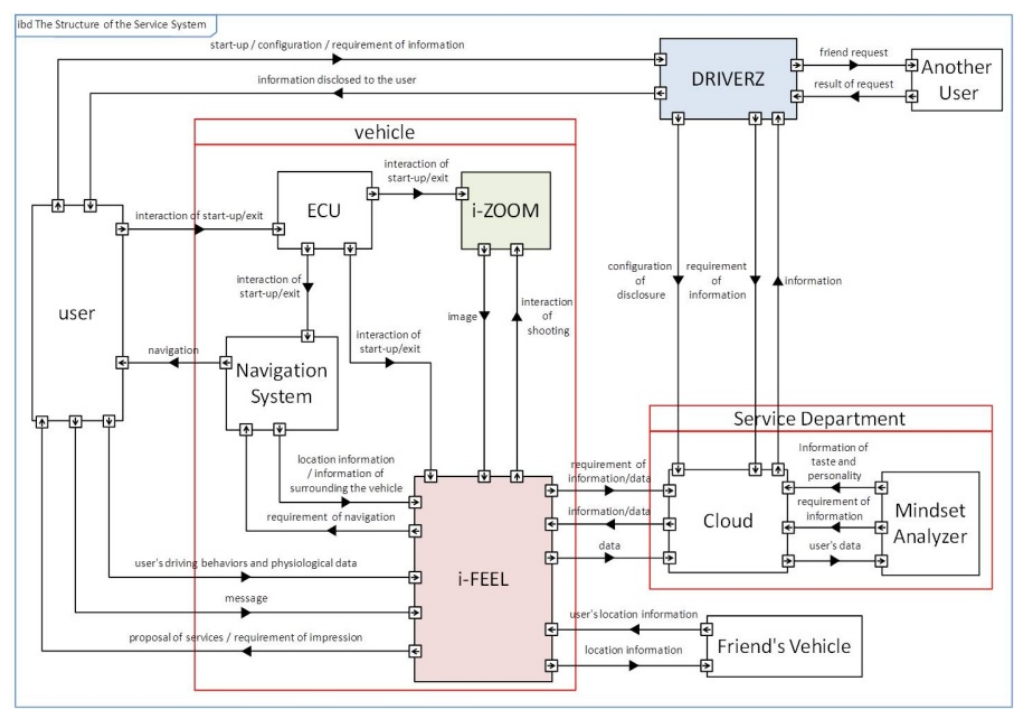

Fig. 6. Internal Block Diagram of the Service System

system. As to main subsystems which encourage the growth of values that user feels towards the mobility, "i-FEEL", "i-ZOOM", and "DRIVERZ" are proposed. First, "iFEEL" is an emotion recognition system carried inside the vehicle. This system recognizes emotions of the user analyzing his behaviors and physiological data, and provides services based on emotions. Secondly, "i-ZOOM" is a camera system carried inside the vehicle. This system safely takes pictures of both the inside and the outside of the vehicle based on directions of the user or cooperation with "i-FEEL" while the user is driving. At last, "DRIVERZ" is a social networking service (SNS) and this system facilitates communication between users through the vehicle by sharing information such as pictures and driving routes. By combining these subsystems, the service system encourages the growth of values that user feels towards the mobility.

\subsection{A Process of Service Delivery to User}

To encourage the growth of values that a user feels towards the mobility, the service system provides services appropriate to relationship between the user and the vehicle with the use of the vehicle. Fig. 7 shows the Value Growth Model and major services in each phase. Along this model, a process of value growth is explained.

In the value discovery phase, the user can get information about mobility system including the vehicle and the service system. Additionally, the user can interact with other users owning vehicle by making friends in DRIVERZ. By these features, the user receives the delight through travel with the vehicle and discovers fascination.

In the value realization phase, the user then begin realizing values of mobility through usage of the mobility system. For example, by combining i-FEEL and iZOOM, i-ZOOM can take pictures of both the inside and the outside of the vehicle when the user enjoys. The service system provides interactive services between the 


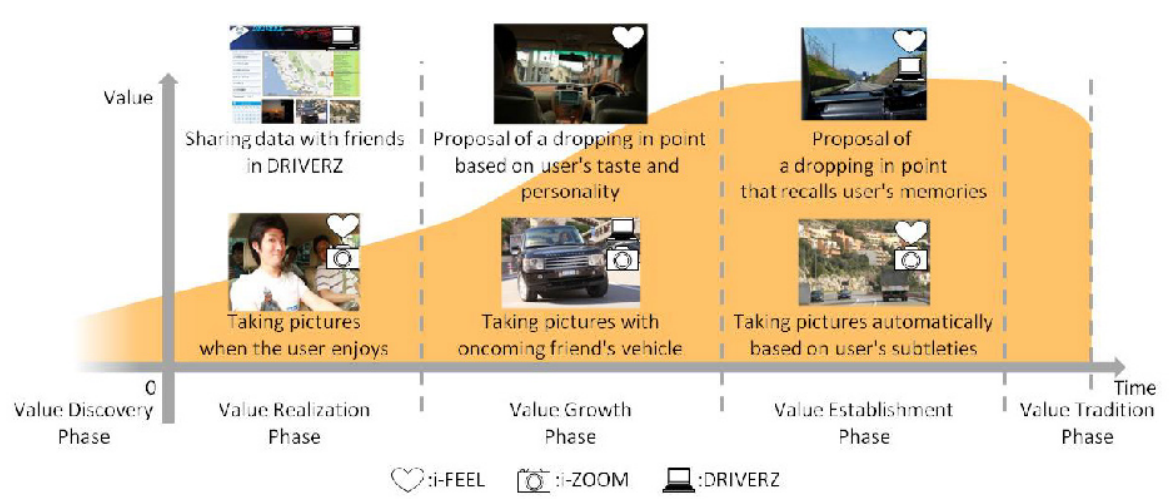

Fig. 7. Images of Services

user and the vehicle by the application of emotion recognition technology. Pictures taken while driving and information of driving routes are stored in cloud computing. The user can manage these data in DRIVERZ, such as browsing and editing data, or sharing data with friends. By combining the vehicle and DRIVERZ, the service system enhances interaction with other users more than before the user purchases the vehicle. Through these function, the user feels the delight through travel with the vehicle and realizes values of mobility.

In the value growth phase, values that the user feels towards the mobility grow the most. Based on the user's data stored in the cloud computing including user's feeling recognized by i-FEEL and sites that user browsed in DRIVERZ, the service system analyzes user's mindset, and delivers services according to them. For example, in the case there is a site around a drive route where the user would be interested, i-FEEL proposes the site as a point of dropping in. If the user decides to drop in the point and conveys the decision to i-FEEL, i-FEEL navigates the user to the point. This information of interaction between the user and i-FEEL through services are stored in the cloud computing, and used to improve analysis of user's mindset. In addition, by using information of user's friends, the service system takes a picture of the user's vehicle and the friend's vehicle when they pass each other. This function promotes interaction of users while driving. In this phase, the user detects to feel various delights by driving the vehicle. Thus, values grow in this phase.

In the value establishment phase, values that the user feels towards the mobility become stable. In this phase, because accuracy of analysis about user's mindset is high, i-FEEL delivers services catching user's subtleties of feeling. For example, when the vehicle finds a view matching user's mindset, the vehicle automatically takes a picture of it without user's directions. Furthermore, the service system becomes capable of delivering proposals that make the user recall memories stored through interactions with the mobility system. For instance, when the vehicle approaches a point visited in past, i-FEEL proposes to drop in the point with pictures at the present time. In this phase, because the mobility system gives these delights through travel with the vehicle, the service system contributes to developing a sense of attachment in the user. Thereby, the user gets motivated drive the vehicle further. 
In the value traditional phase, the user renews the vehicle. The data stored in the cloud computing and data of user's mindset analyzed by the service department are continuously used to deliver services after the user renews the vehicle. Therefore, the user can interact with a new vehicle in a similar way as the vehicle that the user used. Hence, values that the user feels towards the mobility are inherited after the user renews the vehicle.

These services are described in detail by diagrams representing behavior in SysML, and essential systems to realize services are derived. Fig. 8 shows one of sequence diagrams. Sequence diagram represents behavior in terms of a sequence of messages exchanged between parts. This diagram enables to specify the interaction between the user and the mobility system. Time proceeds vertically down the diagram. Fig. 8 is an example of the sequence diagram specifying a behavior "the vehicle recognizes user's emotion". First, i-FEEL obtains user's driving behaviors and physiological data, and analyzes this data and recognizes user's emotion. Then, i-FEEL stores user's emotion and elements causing it in the system and the cloud computing. By doing this, the service system enables to recognize user's emotion and to deliver services based on the particular emotion. In addition, data stored in i-FEEL and the cloud computing are used to improve accuracy of the emotion recognition, as well as to analyze user's mindset. For these reason, it is found that i-FEEL requires a database to store user's data. Thereby, systems that construct the service system are derived by the analysis of behaviors.

\subsection{Provision of Services to Realize Delights through Travel with the Vehicle}

As described in earlier sections, items that construct the service system and services that are provided by these items are assumed to support growth of values that the user

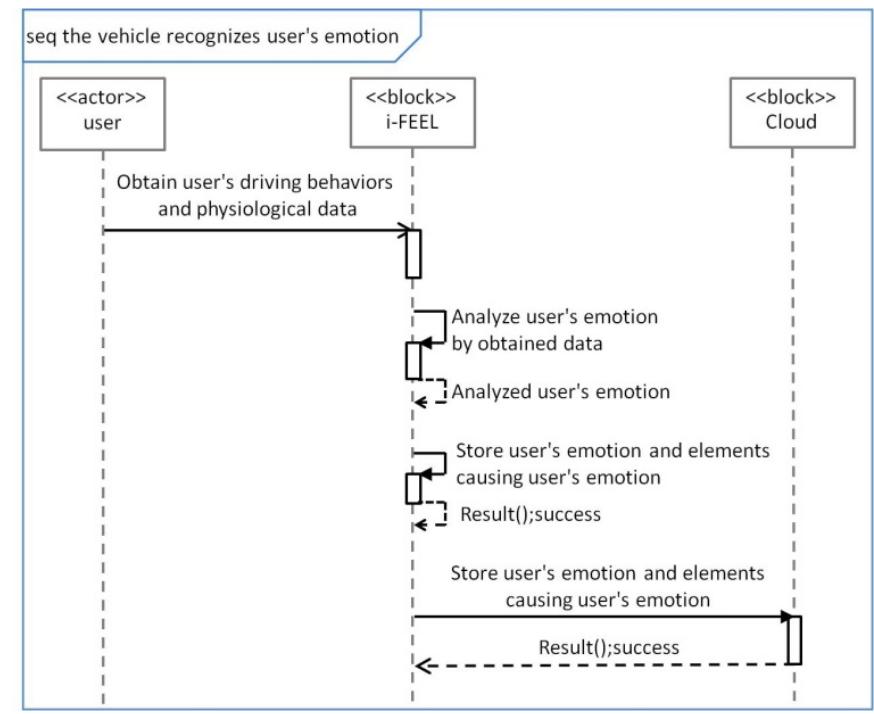

Fig. 8. Sequence Diagram “The Vehicle Recognizes User's Emotion” 


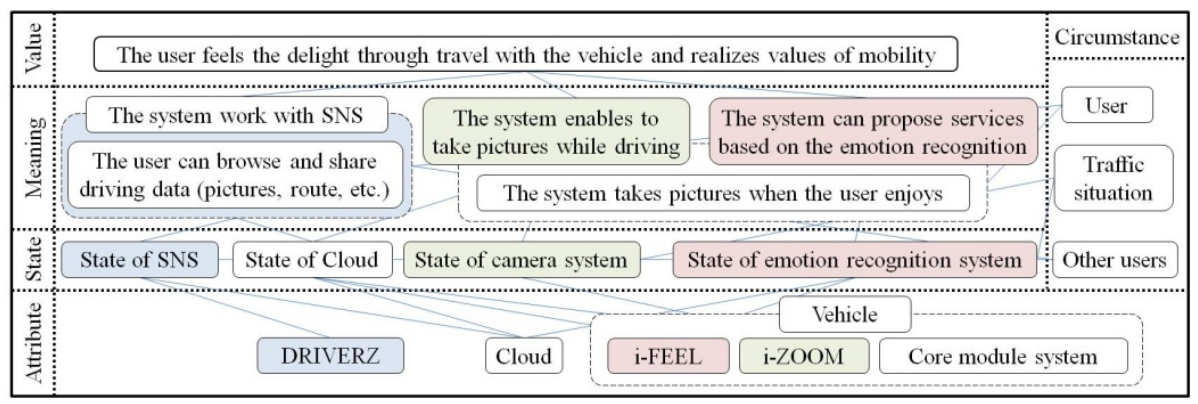

Fig. 9. A Part of Elements Relationship Diagram

feels towards the mobility. Fig. 9 shows a part of an elements relationship diagram arranged architecture of the service system. This diagram describes psychological elements that the user feels towards the mobility and physical elements that realize psychological elements in the value realization phase. Subsystems of the service system including "i-FEEL", "i-ZOOM", and "DRIVERZ" are described as attribute elements. These attribute elements realize state elements, and state elements realize meaning elements such as "the vehicle automatically takes pictures which the user wants" and "the user shares pictures with other users in SNS". These elements satisfy a value element "the user feels the delight through travel with the vehicle". Thus, the service system promotes interaction between the user and the mobility system through SNS not only when the user drives the vehicle, but also when the user is not driving the vehicle. As the result of the interaction, the user feels the delight through travel with the vehicle by using the service system, and this system encourages the growth of values that the user feels towards the mobility.

\subsection{Future Tasks towards Embodiment the Service System}

Towards the realization of the proposed service system, a design of the lowest configuration items is currently developed. Drive recording technology, which is already been commercialized, enable to realize i-ZOOM. Additionally, DRIVERZ can be realized by technologies used in existing SNS to architect software and usage of internet. Similarly, systems generated based on technology seeds are realized by using attribute elements, which are bases of the idea generation, as the lowest configuration items or directly. By contrast, the emotion recognition system that is the core of iFEEL is difficult to realize by using seeds of technology, because technologies of emotion recognition are in the process of development. Thus, it is necessary to consider in detail about index parameters and methods for emotion recognition based on existing technology. As these index parameters, user's behaviors and physiological data are proposed. However, this system has not been developed in detail for embodiment. As a future task, it is necessary to verify feasibility of emotion recognition based on proposed index parameters. As the result of system verification, the lowest configuration items of the emotion recognition system can be decided. Additionally, it is necessary to define items of other systems and develop the design of the service system towards validation, whether it encourages the value growth of mobility or not. 


\section{Conclusion}

In this research, the design of the service system realizing the Value Growth Mobility is developed by the system design method based on the M-V model. As the result of the design, the service system consisting of subsystems including i-FEEL as an emotion recognition system, i-ZOOM as a camera system, and DRIVERZ as a social networking service is proposed. This service system enables to provide interactive services between the user and the mobility system or other users, such as taking pictures automatically based on the user's emotion and sharing information with friends through SNS. By these interactions, it is expected to encourage the growth of values that the user feels towards the mobility.

In further research, toward a validation whether the proposed service system encourages the growth of values of mobility, it is necessary to continue the design of the service system.

\section{References}

1. Mitsubishi Research Institute, Inc., The Grand Design for The New Century, Nikkei Inc. (2000) (in Japanese)

2. Tanaka, S.: Mirai yosoku report 2009-2015, Nikkei Business Publications, Inc. (2008) (in Japanese)

3. Matsuoka, Y.: Dawn of Timeaxis Design, pp. 46-72, 95-104. Maruzen Publishing Co., Ltd. (2012) (in Japanese)

4. Kanazawa, S., Sato, K., Matsuoka, Y.: Value Growth Design Model Based on Design Science. In: The 15th International Conference on Machine Design and Production, UMTIK, Turkey (2012) [CD-ROM]

5. Kamiya, K., Kito, A., Alvarez, J., Sato, K., Nishimura, H., Matsuoka, Y., Furugori, S.: Design of a Service System for Vehicle Users by Applying Multispace Design Method and SysML. In: Proceedings of the Asia-Pacific Council on Systems Engineering Conference 2013, APCOSEC, Japan (2013) [CD-ROM]

6. Furugori, S., Yamazaki, T., Kuroda, Y., Suetomi, T., Nouzawa, T., Ujiie, Y., Nakazawa, K., Matsuoka, Y.: Value Growth Design in a Next Generation Mobility. Oukan, The Journal of Transdisciplinary Federation of Science and Technology 6(1) (2012) (in Japanese)

7. Kawamoto, T.: Vision of Future Automobile. In: JIDOSHA-GIJUTSU. Journal of Society of Automotive Engineers of Japan 67(1) (2013) (in Japanese)

8. Matsuoka, Y.: Multispace Design Model Towards Integration between Industrial Design and Engineering Design. In: Proceedings of Design Research Society 2012. Design Research Society, Thailand (2012)

9. Forsberg, K., Mooz, H., Cotterman, H.: Visualizing Project Management: Models and Frameworks for Mastering, 3rd edn. John Wiley \& Sons, Inc., New Jersey (2005)

10. Matsuoka, Y., Ujiie, Y., Asanuma, T., Takano, S., Izu, Y., Sato, K., Kato, T.: M method Design Thinking on Multispace. Kindai-Kagaku-Sha, Tokyo (2013)

11. Friedenthal, S., Moore, A., Steiner, R.: A Practical Guide to SysML. The Morgan Kauffman OMG Press (2008) 